\title{
Germanica
}

\section{Impossible enracinement, impossible utopie : l'aporie de l'ailleurs dans la trilogie autobiographique de Franz Innerhofer}

Unmögliche Verwurzelung, unmögliche Utopie: die Aporie des Woanders in Franz Innerhofers autobiographischer Trilogie Impossible deep-rootedness, impossible utopia: the aporia of the distance in Franz Innerhofer's autobiographical trilogy.

\section{Evelyne Jacquelin}

\section{OpenEdition \\ Journals}

Édition électronique

URL : http://journals.openedition.org/germanica/254

DOI : 10.4000/germanica.254

ISSN : 2107-0784

\section{Éditeur}

Université de Lille

Édition imprimée

Date de publication : 1 juin 2007

Pagination : 39-57

ISBN : 2-913857-19-1

ISSN : 0984-2632

\section{Référence électronique}

Evelyne Jacquelin, «Impossible enracinement, impossible utopie : I'aporie de l'ailleurs dans la trilogie autobiographique de Franz Innerhofer », Germanica [En ligne], 40 | 2007, mis en ligne le 16 février 2010, consulté le 06 octobre 2020. URL : http://journals.openedition.org/germanica/254 ; DOI : https:// doi.org/10.4000/germanica.254

Ce document a été généré automatiquement le 6 octobre 2020.

(c) Tous droits réservés 


\section{Impossible enracinement, impossible utopie : l'aporie de l'ailleurs dans la trilogie autobiographique de Franz Innerhofer}

Unmögliche Verwurzelung, unmögliche Utopie: die Aporie des Woanders in Franz Innerhofers autobiographischer Trilogie Impossible deep-rootedness, impossible utopia: the aporia of the distance in Franz Innerhofer's autobiographical trilogy.

\section{Evelyne Jacquelin}

Entre 1974 et 1977, Franz Innerhofer, écrivain autrichien né en mai 1944, publie trois romans formant une trilogie autobiographique: Schöne Tage, Schattseite et Die großen Wörter'. Le déroulement de l'action dans chacune des œuvres comme au fil de leur succession suit d'assez près la vie de l'auteur, à travers les vicissitudes que rencontre un alter ego nommé Franz Holl. Le premier volet est consacré à la rude enfance du petit bâtard corvéable à merci dans la ferme de son père durant les années cinquante; le second roman concerne ses années d'apprentissage autant professionnel que personnel entre 1961 et 1963 dans la famille de Joseph Bruckmann, maitre forgeron; le troisième volume narre son expérience de la condition ouvrière en même temps que sa formation intellectuelle sur les bancs des cours du soir, puis de l'université, à Salzbourg vers la fin des années soixante. Cette trilogie est souvent considérée comme l'un des modèles de référence de la Antiheimatliteratur ${ }^{2}$, courant inversant les codes du roman de terroir pour dénoncer après la Seconde Guerre mondiale une idylle désormais frelatée, compromise avec l'idéologie « de la terre et du sang » et pourtant parfois requise à des fins patriotiques par une Seconde République autrichienne qui doit asseoir sa nouvelle identité démocratique après la période de l'austro-fascisme et celle de l'Anschlu $\beta^{3}$. 
Dénonçant une peinture bucolique de la terre natale dont l'idéalisation tient du mensonge historique, c'est la vision de l'ici que cette littérature met en question, par une critique souvent radicale pouvant s'ouvrir sur la perspective d'un ailleurs au moins mental, sinon géographique - songeons par exemple à la tension entre un génie philosophique, théâtral ou musical au demeurant non dépourvu d'ambivalence et l'abrutissement qui caractéristique jusqu'à la caricature la province autrichienne dans l'univers de Thomas Bernhard, ou encore au contraste entre les souvenirs de Carinthie et le voyage vers Rome ou l'Inde pour Josef Winkler.

\section{Exilé dans l'ici}

Chez Franz Innerhofer comme chez beaucoup de ses confrères, l'ici exécré est d'abord un lieu de confinement dont l'étroitesse exclut précisément l'ailleurs. Les déplacements géographiques décrits dans la trilogie en témoignent : on ne quitte guère la région de Salzbourg au fil de ces pages qui mènent seulement de la haute vallée de la Salzach, austère théâtre d'une enfance et d'une adolescence livrées à la brutalité dans Schöne Tage et Schattseite, à l'étouffante ville des princes-archevêques où se déroule Die großen Wörter. Si ce dernier roman offre quelques échappées géographiques, c'est justement parce que le héros est devenu un jeune homme révolté que son désir d'émancipation mène vers des destinations revêtant une forte valeur symbolique : le Paris libertaire des années soixante-huit et cette Italie qui deviendra de plus en plus, pour l'auteur, l'envers paradisiaque d'une Autriche en reconstruction, endurcie dans l'acharnement au travail et frappée de vénalité avec la reprise des affaires et l'essor du tourisme.

Mais ces ouvertures sont tardives et éphémères: dans son ensemble, la trilogie d'Innerhofer se joue dans les limites étroites de cette province salzbourgeoise où le repli géographique se double pour Franz Holl d'une situation d'aliénation sociale et psychologique qui ôte tout caractère familier au milieu dans lequel il se trouve plongé par sa naissance. Rejet de l'ici suscitant l'émergence d'un autre horizon, ce dispositif se complique donc du fait que l'environnement immédiat, plein d'une violence soustraite à toute explication, est lui-même vécu comme radicalement étranger. Franz Holl se trouve d'abord marginalisé, dès sa naissance, parce qu'il est le fruit d'amours illégitimes entre Rudolf Lein, paysan indépendant, propriétaire de son bien, et une servante qui trouvera à se marier bientôt avec un aussi pauvre qu'elle. Dans cette Autriche rurale et profondément catholique bouleversée par les années de guerre, le bâtard n'est pas exclu, mais relégué sans qu'on lui assigne de place fixe. Ainsi, Holl est ballotté entre les diverses maisons où il passe successivement ses toutes premières années - famille d'accueil, ferme de sa grand-mère maternelle, puis de sa mère et de son beau-père - avant d'être officiellement placé comme semi-orphelin chez son père naturel, près du village de Haudorf. Mais il se trouve également pris entre les fronts sociaux puisque même privé de tout droit à héritage, il demeure toujours, dans un premier temps, fils de patron aux yeux des ouvriers agricoles dont il partage pourtant le sort. Par ailleurs, il est définitivement marqué, au sein de la communauté villageoise, par l'opprobre lié à sa naissance comme en témoignent les premières scènes d'église montrant la condamnation répétée de sa mère par le prêtre de la paroisse ${ }^{4}$. Son père le désignera du reste souvent, par la suite, comme son "péché de jeunesse ${ }^{5}$, preuve vivante d'une virilité qui triomphe jusque dans ses écarts tandis que la honte pèse à jamais sur la femme et l'enfant. 
4 Au-delà de ces aspects sociaux, c'est le dispositif narratif choisi par Franz Innerhofer et son écriture qui lui permettent d'inverser le thème de la Heimat pour en faire le lieu d'un exil essentiel, inhérent à la condition marginale de Holl et de ses semblables, au nom desquels l'auteur entend aussi parler ${ }^{6}$. La première phrase de Schöne Tage, souvent citée, substitue au motif de l'attachement à la terre natale celui de l'arrachement qui jette le personnage central dans un monde étranger :

Der Pflege einer kinderlosen Frau entrissen, sah Holl sich plötzlich in eine fremde Welt gestellt?.

Dans ce passage inaugural, la focalisation du récit sur le tout jeune enfant renforce cette inversion de l'ici familier en un ailleurs inquiétant selon un dispositif qui marque l'ensemble de la trilogie. Holl naît et grandit dans un monde où l'usage de la parole se trouve réduit à l'énoncé de commandements, qu'il s'agisse du travail à effectuer ou du rappel aux normes morales ${ }^{8}$. La langue raréfiée, souvent remplacée par des gestes coups portés contre l'enfant récalcitrant, "regards annihilants» des femmes qui condamnent toute transgression ${ }^{9}$ - ne sert jamais à éclairer un univers où l'individu pourrait trouver une place qui lui convienne, mais impose une soumission muette à un ordre légitimé sans explication. Et l'inconcevable brutalité de ce «monde de heurts et de coups $»^{10}$ est particulièrement mise en relief dans la mesure où elle est décrite du point de vue de l'enfant abandonné à sa solitude, exposé sans moyen de comprendre. La violence mine et dévoie l'espace familier qui se réduit dès lors au voisinage immédiat du corps : Holl se réfugie ainsi derrière la table de la cuisine qui lui sert de Heimat, chez sa grand-mère, et le monde extérieur devient chez sa mère une " terre étrangère » où il se sent envoyé chaque jour comme le soldat d'une armée d'invasion ${ }^{11}$.

L'arrachement et la confrontation brutale avec un monde dont l'hostilité demeure inexpliquée constituent une expérience récurrente au cours des premières années de Holl. À six ans, il a donc connu quatre maisons différentes où il est arrivé chaque fois comme le fruit du péché, dans la même stupeur muette. Et à la ferme paternelle, où il va passer les onze années suivantes, les changements de commis et de filles de ferme, tous les ans à la Chandeleur, prolongeront cette souffrance : l'enfant les vit comme un cataclysme réitéré où s'engloutit le peu de familiarité qu'il a pu chaque fois construire, ce qui le rejette sans cesse dans l'inconnu ${ }^{12}$. Cette répétition fait du sentiment d'exil un mode d'être, et de l'ici un ailleurs permanent.

\section{L'ailleurs : une quête perpétuelle}

7 Si Holl subit dans la violence une marginalisation qui fait de son voisinage immédiat, jusque dans la sphère intime de la famille, un espace étranger et menaçant, il va s'approprier cette situation en transformant la semi-exclusion qui lui est imposée en refus d'appartenance. Son père est ainsi rarement nommé ou évoqué comme tel : sa fonction, ramenée à une dimension purement biologique à travers le terme de géniteur, Erzeuger, voire assimilée au rôle de l'ennemi par excellence, Widersacher ${ }^{13}$, est plus souvent escamotée par une dénomination renvoyant au seul statut social - Rudolf Lein est avant tout der Bauer, c'est-à-dire le patron, terme par lequel Holl se place, lui, du côté des ouvriers agricoles. Au fil du temps, le fossé se creuse entre le personnage central et un environnement qui provoque en lui une nausée existentielle, un dégoût absolu du monde et de lui-même. Il s'agit d'abord d'un sentiment déclenché par les punitions injustes et brutales qu'il doit sans cesse subir ${ }^{14}$, mais un retournement 
s'opère à l'occasion d'une fugue ratée suivie d'une nouvelle pénitence au cours de laquelle l'écœurement se double de haine et devient une première forme d'opposition désespérée :

Mit der Sonne stiegen auch die Bitterkeit und der Ekel. Er haßte dieses Tal und die Menschen rundherum so sehr, dass er auf einmal keine Angst mehr hatte.

[...] Da war ja alles so schön, so paradiesisch falsch, daß ein Schlag ins Gesicht nur noch alles verschönerte. Man lachte einfach mit [...]. Das Dorf war schon so ungeheuer fremd. [...] Man lernte sparsam mit der Hoffnung umzugehen. Man war in der Fremde beschäftigt mit der Fremde.

Das war Autodidaktik.

Was einer für den anderen tun konnte, war nicht viel. [...] Wer davon laufen konnte, lief davon. Viele standen es durch, bis sie ihre Situation erkannten, dann brachten sie sich um. Es hieß dann einfach : Der oder die HAT SCHLUSS GEMACHT. [...] Es klang wie eine Billigung, als ob man von denen, die von der letzten Möglichkeit Gebrauch machten, nichts anderes erwartet hätte. Selbstmord war und ist für diese Leute Übereinstimmung ${ }^{15}$.

Ce qui se révèle dans la violence répétée des châtiments immérités, c'est que l'ici est inhabitable, parce qu'inhumain. Au-delà du rire amer se dessine alors la recherche encore obscure d'une échappée autre que le suicide, puisque celui-ci ne sert finalement qu'à conforter cet enfer. La suite de la trilogie est le récit de cette révolte incertaine, toujours menacée, dont le premier pas est la décision de ne rien partager avec un univers désespérant de brutalité. Le très long passage consacré à la onzième année de Holl, qui couvre pratiquement un tiers de Schöne Tage, débute entre autres par la réponse que se fait intérieurement l'enfant auquel on ne manque jamais de rappeler sa position de bâtard, en marge de la famille légitime : «Er wollte ja gar nicht zur Familie gehören. $»^{16}$

La difficulté de ce divorce réside dans le fait que le personnage n'a aucun repère lui permettant d'imaginer un autre ailleurs que cet environnement étranger et hostile. Les valets qui changent de patron chaque année ne font en fait que tourner en rond, pouvant au mieux tenter leur chance comme manœuvre ou bûcheron afin de fuir leur condition $^{17}$. Pour échapper à cet enlisement, il faut une intervention extérieure : ce sera une femme étrangère au village, une aide-cuisinière venue de la ville, lisant les journaux et recevant du courrier de divers pays, qui va mettre en question de manière radicale l'évidence d'un ordre dont l'immuable brutalité passe pour naturelle et intangible. À ce premier stade, le nouvel horizon qui s'ouvre pour Holl ne correspond donc pas à un départ réel, mais à une manière différente de penser l'ici : l'arrivée de Helga le bouleverse en lui montrant qu'il existe une autre façon d'être au monde, hors de la violence dans laquelle il baigne depuis sa naissance. S'opposant par la parole et le geste à la marche des choses, cette servante singulière fait la preuve qu'il est possible à la fois de résister et de se mettre à l'écoute de l'autre. Elle est en particulier la première à voir en Holl non le rejeton un peu raté que sa sourde opposition fait passer pour sournois, mais l'enfant abruti de travail, rendu incontinent par l'état d'abandon et de terreur dans lequel il grandit, dont on raconte les prétendus méfaits en sa présence " comme s'il n'était qu'un tas de pierre ${ }^{18}$.

10 La voie ouverte par Helga sera celle d'une rébellion toujours plus franche, qui, d'abord instinctive, va se structurer peu à peu pour se muer en une critique sociale d'inspiration marxiste au cours des deux romans suivants. Ce développement s'amorce dès l'adolescence de Holl, dans Schöne Tage, où le thème de l'aliénation par le travail revient de plus en plus souvent à mesure que le personnage central grandit et prend 
conscience du monde. Le jeune garçon se sent ainsi réduit à un simple corps, force de travail exploitée sans vergogne par son père ${ }^{19}$. Après l'aide-cuisinière, Hermann Klein, un trayeur embauché au tout début des années soixante, alors que Holl a passé seize ans, saura exprimer cette critique en termes plus immédiatement politiques ${ }^{20}$. Mais le bâtard se trouve en porte-à-faux : attaché à la ferme comme un serf ${ }^{21}$ par des liens du sang qui ne lui donnent aucune prérogative, il ne peut même pas réclamer les droits que les ouvriers agricoles vont peu à peu faire valoir ; par ailleurs, n'ayant ni formation ni métier, il est condamné à cet esclavage sans autre avenir qu'être harassé de travail jusqu'à la mort comme le vieux Moritz en lequel il reconnaît une destinée qu'il veut fuir à tout prix :

Der Bauer habe sich einfach Holls Körper angeeignet, wie sich der Großvater den Körper von Moritz angeeignet hatte. Der Kopf von Holl sah den Körper von Moritz immer tiefer in die Knie gehen und hörte, dass ihnen der Körper von Moritz nicht mehr passte. Ein wandelndes Marterl, das man loshaben wollte. Ein Leben lang im Bauern-KZ, aber nun nur mehr Haut und Knochen, die Speisen gingen ihm halbverdaut in die $\mathrm{Hose}^{22}$.

11 L'appel de l'ailleurs, dans cette perspective, revêt plusieurs aspects. Dans le monde confiné de la ferme où Holl entame son adolescence sans perspective ni moyens d'action, la critique de l'ici ne peut mener qu'à la fuite. Il lui faut s'arracher à une terre natale où il n'a pas de place pour échapper à la condition de paria qui lui est faite. Seul le hasard rendra la chose possible: Holl est pris comme apprenti par Joseph Bruckmann, maître forgeron au bourg de Haudorf, son meilleur ami ayant renoncé à cette place au dernier moment. Schöne Tage se termine ainsi par une sorte d'heureuxdénouement qui entraîne le personnage principal vers un « monde nouveau » enfin accueillant: dans la maison des Bruckmann, les visages sont "étrangers et amicaux $\aleph^{23}$ et Holl accède à une forme plus digne d'existence alors qu'on lui accorde l'intimité d'une chambre et qu'on le considère comme un véritable interlocuteur, éveil résumé en une formule :

Hier arbeitete er ja nicht für besitzsüchtige Bestien, sondern mit Menschen, nach mehr als siebzehn Jahren war er plötzlich unter Menschen ${ }^{24}$.

12 Si l'on ne pousse pas plus avant la lecture de la trilogie, s'arrêtant au premier roman qui demeure aussi le plus célèbre, on pourrait croire qu'Innerhofer limite sa critique de la société autrichienne à la dénonciation du patriarcat rural tel qu'il existait au sein d'une économie encore peu mécanisée, et ce dans le contexte de pénurie de l'immédiate après-guerre - ordre dont la disparition se dessine à la fin du roman avec l'arrivée des machines agricoles et le pouvoir qu'elles donnent soudain à Holl, seul capable d'en assurer la maintenance. La dimension autobiographique de l'œuvre semble conforter cette vision, l'achèvement du roman pouvant faire office de preuve pour démontrer que l'Autriche moderne permet au plus défavorisé de ses enfants de trouver le chemin du savoir, de la culture et même du succès littéraire. L'ailleurs infernal qu'était l'ici serait dès lors dépassé par les progrès de l'histoire, laissant place à un monde enfin habitable, avènement de cet autre ailleurs à peine imaginable lorsque Holl cherchait à s'échapper.

13 Mais l'expérience de l'exil au sein même de l'ici n'est pas seulement un mode de vie passif; elle génère aussi une manière particulière d'appréhender le monde. Holl y pose le regard de celui qui n'est jamais chez lui, n'a pas de repères fixes ni de certitudes et doit sans cesse recomposer sa vision des choses: quel que soit le nouveau milieu où il tente de faire son chemin, il se sent à jamais en décalage avec les gens installés, ce qui 
va aiguiser un sens critique auquel l'initie par ailleurs la mère de son nouveau patron, Helene. Schattseite et Die großen Wörter, loin de présenter le récit apaisé d'une ascension sociale dans un environnement enfin humain, renouvellent au contraire les attaques d'Innerhofer, mettant en question certains fondements de la société autrichienne telle qu'elle se reconstruit après la guerre, notamment la modernisation économique, avec la mécanisation de l'agriculture, le développement de l'automobile ou l'essor du tourisme, ainsi que la démocratisation de l'enseignement et de la culture ${ }^{25}$. Même si la brutalité en est atténuée, l'ici demeure donc un lieu dont le personnage ne peut s'accommoder : il continue dès lors à vouloir toujours fuir pour un ailleurs qu'il se met à construire en découvrant le monde, en particulier à travers les lectures et les films auxquels il s'ouvre peu à peu sous la conduite de Helene ${ }^{26}$.

\section{L'ailleurs comme instrument critique}

Holl arrive chez les Bruckmann l'esprit encore fruste et plein de craintes. Aiguillonné par Helene, il va reconnaître son ignorance et accepter de la surmonter. Les premiers livres qu'il consulte illustrent avec précision le rapport dialectique qu'entretiennent l'ici et l'ailleurs aux yeux du jeune homme incapable de compromis avec le monde qui l'entoure. Les contrées lointaines sont avant tout convoquées pour conforter son refus de la terre natale, de la Heimat, exécration qui ne se résout pas en une fuite vers ces horizons peut-être accueillants, mais débouche sur un questionnement appelé à devenir de plus en plus nettement politique et social. Ainsi, le premier ouvrage tombant entre les mains de Holl est un atlas qui le fascine d'abord par tout ce qu'il recèle d'inconnu, mais le ramène bientôt à une réflexion critique sur l'Autriche et sur sa propre place au sein de son pays :

Im Zimmer fand ich unter Büchern einen Atlas, blätterte, suchte nach Ortsnamen.

[...] Stieß ich beim Blättern auf eine Österreichkarte, blätterte ich schnell um.

Österreich interessierte mich überhaupt nicht. Das Wort Österreich war mir peinlich. Ich lernte die Namen ausländischer Hauptstädte und zählte sie auf, um keinen österreichischen aufzählen zu müssen. [...] Ich weigerte mich lange, Österreich in Bundesländer einzuteilen. 8 Bundesländer, sagte ich mir.

Das ist doch der Mühe wert, daß man so ein kleines Land in acht Bundesländer einteilt. Noch weniger wollte ich natürlich wahrhaben, dass es 10 Regierungen gibt. [...] 8 Bundesländer, 10 Regierungen, darunter eine Große, und alle Regierungsmitglieder, ihre Frauen, ihre Kinder müssen Geld bekommen [...]. An diese Leute hatte ich vorher kaum gedacht [...]. Helene sagte, es ist wichtig zu wissen, wer regiert. [...]

Den Atlas legte ich bald zur Seite.

Bücher fingen an, mich zu interessieren.

Ich wollte nun selber mehr wissen. Die Frage nach meiner Zugehörigkeit wurde mir immer rätselhafter ${ }^{27}$.

Les livres qui vont permettre à Holl de clarifier peu à peu la manière dont il se situe dans le monde s'inscrivent dans ce même mouvement d'appel vers l'ailleurs pour mieux mettre en question l'ici. Le jeune homme rend ainsi à Helene un roman paysan qu'il juge, en connaisseur du milieu, parfaitement mensonger - dans une inscription métatextuelle du projet même de l'auteur - avant de faire pour longtemps ses délices d'une biographie de Napoléon. Ce n'est pas tant l'histoire de France, la Révolution ou l'Empire qui le fascinent dans cet ouvrage, mais bien davantage le contraste avec son propre environnement. L'extraordinaire liberté dont jouissent alors les classes 
privilégiées, le monde des salons, avec tout son apparat, son luxe de toilettes et de bijoux, enflamment l'imagination de l'adolescent. Cependant, loin de se contenter d'une échappée factice dans des fastes et un décorum surannés, il en retient avant tout qu'une autre vie est possible. Et les questions qui surgissent alors de manière encore générale et maladroite sont celles-là mêmes qui vont former les axes principaux de sa réflexion politique ultérieure: la place du travail et la place des mots dans une existence humaine. Lui qui vit dans un univers où il y a toujours à faire, où l'on s'échine sans repos, est d'abord frappé par le loisir dont disposent les personnages rencontrés au fil des pages, au point qu'il n'y a pas pour eux de « distinction entre jours ouvrés et jours fériés, mais simplement des jours, du temps $\aleph^{28}$. Par ailleurs, il note l'importance des «belles et longues conversations » touchant à des sujets dont il s'étonne qu'on puisse même les aborder alors que toute tentative pour parler de soi, de ses peurs et de ses difficultés, étaient assimilées dans son enfance à autant de jérémiades indignes d'ancêtres rudes à la peine, injonction au silence revenant à confiner les êtres dans leur solitude au lieu de les laisser s'ouvrir à la vie en communautée 29 .

16 Ayant échappé à l'isolement mental dans lequel il était enfermé chez son père, Holl va pouvoir commencer un véritable apprentissage intellectuel auprès des Bruckmann en même temps qu'il se forme au travail du métal. Ce séjour de quelques années seulement, raconté dans Schattseite, présente bien plus de contradictions que la vie à la ferme. Durant la première enfance de Holl, l'ici, entièrement placé sous le signe d'une brutalité sans paroles, ne pouvait donner lieu qu'à la détestation la plus complète et, finalement, à la fuite, sous quelque forme que ce soit. Dans cette famille étrangère en revanche, Holl arrive enfin en territoire humain : on aurait pu s'attendre à ce qu'il y trouve sa place et s'y enracine, mais on a vu qu'au contraire, le sentiment d'exil génère une mise en question perpétuelle de l'ici qui laisse le personnage sans repos, d'autant que la violence ne disparaît évidemment pas du monde. Les expériences nouvelles qu'en fait Holl, notamment sous la férule d'un professeur extrêmement brutal à l'école de formation professionnelle, mais aussi parmi les jeunes ouvriers de Haudorf qui n'acceptent pas un fils de paysan dans leurs rangs et le poursuivent de leur haine - tout cela le conforte dans une critique de plus en plus radicale du monde qui l'entoure. Cette opposition d'abord instinctive va trouver sa forme au cours des nombreuses conversations avec Helene et son époux, Georg, cheminot porteur de valeurs ouvriéristes qui détonnent dans le milieu profondément rural et catholique du village. Ces deux figures tutélaires vont en particulier attirer l'attention de Holl sur la nécessité de ne pas se laisser aveugler par une morale du travail masquant des rapports de domination que Franz Innerhofer révèle notamment à travers les portraits d'employés exploités que dresse son personnage principal ${ }^{30}$.

L'ironie de Schattseite réside dans le fait que cet apprentissage de la critique sociale va se retourner contre Joseph Bruckmann, jeune patron pourtant bienveillant avec qui Holl pourrait tisser des liens d'amitié et auprès duquel il lui serait sans doute possible de faire son chemin. La très longue scène de l'excursion improvisée, vers la fin du roman, en témoigne avec éclat. Elle débute par un moment de complicité entre l'apprenti et son maître endetté, qui se cache pour échapper à la visite finalement démentie d'un huissier. La frayeur passée, Bruckmann décide contre toute raison de fermer pour la journée et d'emmener Holl faire un tour dans les montagnes. Mais au lieu d'un rapprochement entre les deux hommes lors d'une excursion qui s'annonce « idyllique ${ }^{31}$, la journée tourne au règlement de compte : obligé de suivre le rythme 
imposé par Bruckmann durant l'ascension, Holl prend une fois de plus conscience avec rage de sa position subordonnée et va refuser, dans la discussion qui s'engage lors de la pause, toute forme de connivence. Même si elle émane d'un d'adolescent écorché, cette réaction a en fait bien peu à voir avec une question de psychologie ou de morale individuelle. Le «fossé " que le jeune homme tente alors de désigner à l'attention de Bruckmann ne sépare pas des êtres singuliers, mais des classes sociales, si bien que l'entrepreneur plaidera sa cause en vain: il ne peut être question, pour l'employé, d'adopter son point de vue ni de compatir à ses difficultés financières ou à ses problèmes de concurrence ${ }^{32}$. Une fois de plus, le projet de Franz Innerhofer s'affiche nettement dans ce retournement de situation: comme le souligne indirectement son alter ego littéraire dans la même scène, la trilogie ne vise pas à retracer un parcours exemplaire permettant de surmonter un handicap social ${ }^{33}$, mais orchestre une fuite en avant au cours de laquelle le questionnement du personnage central rebondit sans cesse pour déboucher sur une mise en cause de la société tout entière. La problématique de l'ailleurs se reformule au gré de cette évolution. Holl va poursuivre sa quête en partant pour Salzbourg, où il entend reprendre des études et une nouvelle fois, l'ailleurs tient moins dans ce changement géographique que dans l'espoir placé en un nouvel horizon humain, celui du «monde de la parole », domaine de la culture, du savoir, de la pensée que Holl imagine différent d'un « monde du travail » disqualifié par des rapports de domination et d'exploitation.

\section{Critique et utopie : l'aporie de l'ailleurs}

18 La question qui émerge au fil des textes en même temps que s'aiguise leur portée critique est celle d'une nouvelle forme de société : pour que l'ici ne soit plus un ailleurs barbare, en deçà de l'humain, il faut tenter d'esquisser les contours d'un autre monde possible, donc emprunter les chemins de l'utopie. Celle-ci apparaît seulement dans le dernier volet de la trilogie, lorsque Holl acquiert assez de bagage intellectuel pour pouvoir formuler un questionnement plus structuré. La dynamique qui fait passer de l'enfermement dans un ici étranger à la recherche d'un ailleurs moins inhospitalier se trouve donc encore à l'œuvre dans Die großen Wörter où elle revêt plus précisément la forme d'une tension entre critique et utopie.

19 À Salzbourg, Holl se trouve confronté à de nouvelles formes de violence sociale, autant dans les milieux de l'éducation et de la culture qu'à l'usine, et en pénétrant lui-même dans la sphère du logos, il s'en approprie peu à peu les armes pour poursuivre l'entreprise de critique systématique amorcée sous la conduite de Helene et Georg. La dénonciation des rapports de travail dans un monde soumis à la loi du profit, telle qu'elle se développait dans Schattseite à travers l'évocation des petits entrepreneurs apparus à la faveur de la modernisation rurale dans les années soixante, s'exacerbe dans Die großen Wörter avec les descriptions de l'usine de mécanique dirigée par Feinschmied à Salzbourg: logements insalubres des ouvriers, ateliers extrêmement bruyants, consignes de sécurité rarement respectées, hiérarchisation stricte entre cols blancs et bleus de chauffe, syndicat aux ordres, escroqueries organisées d'en haut y sont la règle. La charge se généralise du reste pour mettre en cause une logique mercantile accélérée par le développement de la publicité qui transforme l'univers en un vaste ensemble de produits malléables et échangeables à l'infini. La nature ellemême perd l'aura menaçante qu'elle possédait dans le vieux monde rural au sein 
duquel a grandi Holl, maîtrisée et finalement dégradée par la rationalité urbaine et ses calculs $^{34}$.

Cette critique de l'ici touche au monde économique et laisse intact l'ailleurs du logos. Or l'espoir placé par Holl dans ses études et la découverte de la culture va être à son tour détruit par l'expérience : les professeurs n'ont aucun désir de s'adapter à un public défavorisé, ils parviennent au mieux à faire progresser les élèves déjà parfaitement adaptés au système scolaire et social et n'ont d'autre part aucun recul critique en ce qui concerne le passé récent de l'Autriche, notamment marqué par l'austro-fascisme et le nazisme ${ }^{35}$. Loin de coïncider avec ce que Holl en attendait, l'ailleurs de la parole ne se démarque donc pas essentiellement de l'ici sur lequel s'exerce une critique de plus en plus systématique. Au lieu de l'émancipation qu'il s'en promettait, Holl va rencontrer dans ce monde l'indifférence et le mépris ${ }^{36}$. Surtout, il va se rendre compte que la langue dans laquelle, sous l'influence de Helene, il voyait un instrument d'élucidation et une arme contre un isolement mental destructeur, participe elle-même aux mécanismes de domination économique et politique. Le cours de philosophie dispensé à l'université substitue ainsi au Manifeste communiste, étudié seulement pour en détourner les étudiants, une analyse des techniques commerciales les plus novatrices ${ }^{37}$. Et en face d'institutions acquises à cet ici marchand que le personnage condamne sans appel, les intellectuels tentés par la révolution en ces années d'agitation estudiantine n'offrent guère plus de recours : leur engagement se réduit souvent à une simple pose et risque toujours de se dévoyer dans un enrôlement que va dénoncer une figure clef de la fin de la trilogie, Stürzl, défenseur d'une insaisissable utopie symbolisée par un non moins mystérieux " Manifeste de Summerau ", du nom du village où il entend installer le siège du communisme mondial ${ }^{38}$.

21 À travers sa dynamique critique et sa quête d'un ici plus humain, toute la trilogie converge vers cette visée utopique qui en est comme le point de fuite et correspond aussi à l'ultime figure de l'ailleurs. Ainsi mise en perspective, la quête de Holl apparaît comme une série de rebonds entre ici et autre part : il passe du hameau au bourg, puis à la ville, c'est-à-dire de la ferme à l'atelier et à l'usine, mais aussi de l'école communale aux cours du soir et à l'université, ou encore de la condition de commis agricole au statut d'apprenti, puis d'ouvrier et finalement, inscrite en filigrane à travers la dimension autobiographique de la trilogie, à sa propre consécration en tant qu'écrivain. Mais chaque étape se solde par une désillusion, y compris l'entrée dans le monde intellectuel : les possibilités de trouver dans la réalité un ailleurs habitable se trouvent donc épuisées et le dernier élan possible ne peut être, dès lors, que d'ordre spéculatif. Or précisément, Innerhofer n'est pas homme de pures conjectures : posant toujours le problème dans sa dimension concrète, il arrive à un point de non-retour, une forme d'aporie qui fait basculer la fin du dernier roman vers une farce rageuse où l'idée même de projet utopique structuré vole en éclats. Ce dynamitage s'opère sous un double signe, point de rencontre entre un humanisme que l'on pourrait qualifier de radical en ce qu'il accorde une valeur d'absolu à l'expérience vécue et, d'autre part, une réflexion critique sur la langue comme instrument de pouvoir ${ }^{39}$. D'un point de vue concret, le règlement de compte vise en premier lieu le discours politique qui prétend alors mener vers une société meilleure, voire parfaite : le marxisme-léninisme dans la version professée par le parti communiste. L'écrivain s'en est expliqué à l'époque dans une remarque révélant l'impasse où l'a mené la trilogie et le rôle qu'y tient le travail de la langue : 
Eigentlich wollte ich einen positiven marxistischen Ausgang für das Buch. Aber beim Schreiben sind mir plötzlich Sätze eingefallen, daß ich merkte - alles, was ich bisher erlebt habe, hat sich als nichtig herausgestellt. Ich konnte diesen Schluß den Leuten im Buch nicht antun, es wäre eine völlige Lüge gewesen ${ }^{40}$.

Il est important de noter à propos de cet aveu que le mensonge historique est révélé par le travail d'écriture lui-même, qui suppose une appropriation de la langue maniée dans son authenticité et non figée dans des formules préfabriquées - qu'il s'agisse de la sagesse populaire porteuse de résignation propre au vieux monde rural ou de slogans révolutionnaires instrumentalisés. Au-delà de la classique dénonciation du stalinisme et de ses avatars ${ }^{41}$, la critique du parti communiste se fait donc au nom de l'expérience individuelle, concrète, et de sa libre expression dans un discours authentique - souci que l'on retrouve par exemple aussi chez Peter Handke. Dans le long monologue qui clôt Die großen Wörter, Stürzl se place précisément de ce double point de vue. Il défend une humanité non réductible au collectif, position que l'on pourrait rapprocher du personnalisme, et oppose d'autre part, dans sa critique de la langue, le dévoiement que celle-ci subit au service du pouvoir et la recherche d'une authentique adéquation entre les mots et le monde. Il insiste ainsi sur le fait que les étudiants contestataires s'arrêtent à quelques analyses abstraites sans mettre en jeu leur personne dans l'acte critique :

« Das ist ja ganz nett, daß sie alles lesen, was aus Moskau kommt. Was Ost-Berlin und Leipzig schickt, lesen sie alles. Aber sie haben keine Person. Ein Kommunist muß auch zusehen, daß er zu einer Person kommt. Ich bin ja nicht Kommunist geworden », schrie Stürzl [...], «um Kommunist zu sein, einfach weil ich mir gedacht habe, Kommunist wäre noch das Richtigste, sondern weil ich ein Mensch sein möchte und später einmal als Mensch existieren will. Das verstehen die aber nicht, im Gegenteil, schulen möchten sie mich. Umschulen. Auf ihren Bewusstseinzustand herunterschulen. $»^{42}$

Par ailleurs, Stürzl dénonce la manière dont les institutions s'approprient la langue pour en faire un instrument de domination, privant les petites gens de toute expression propre, selon une thématique déjà développée sur un autre plan dans les deux premiers volets de la trilogie au fil des expériences vécues par Holl dans un monde qui le condamnait au mutisme ${ }^{43}$. À cet égard, les révolutionnaires de profession ne diffèrent pas à ses yeux de la société qu'ils dénoncent, mais perpétuent au contraire cette forme d'aliénation linguistique qui empêche l'émergence de tout discours véritablement critique : on ne vit pas si mal de discours révolutionnaires abstraits qui confèrent une autorité confortable ${ }^{44}$.

Passer du côté des "grands mots ", c'est donc prendre le risque d'une trahison qui ne tient pas simplement à un changement de classe sociale, ne se résume pas à l'embourgeoisement souvent moqué des parvenus ou des révolutionnaires assagis, mais qui touche à l'exercice même de la pensée telle qu'elle s'exprime dans le langage. L'exigence qu'y oppose Innerhofer est tragique en ce qu'elle pousse l'utopiste à refuser au bout du compte tout projet structuré dans un discours qui pourrait être une trop pesante clôture. C'est ainsi que peut s'expliquer la fin très étonnante de la trilogie, où le monologue de Stürzl se désarticule et tourne au grotesque en mêlant parodie, ironie cynique, cris et pantomime, finissant par engloutir son "Manifeste » dans une suite $d^{\prime}$ 'hypothèses contradictoires ${ }^{45}$. Le personnage disparait au paragraphe suivant, mort dans des circonstances masquées par une audacieuse ellipse du texte qui oblige le lecteur irrité à se faire détective pour traquer ligne à ligne une possible explication et découvrir que Stürzl a dû périr au moment où finissait de se désagréger ce long 
discours adressé à Holl, auquel il laisse en testament l'injonction qui clôt la trilogie de ne produire que "des phrases vécues». Avec le "Manifeste» escamoté, ce final au rythme furieux et syncopé détruit l'ailleurs utopique qu'a poursuivi Holl durant toute la trilogie, faisant de lui un personnage sans lieu, à jamais exilé dans la souffrance du divorce entre critique et utopie. Mais on peut aussi penser que cet ailleurs utopique renaît de ses cendres sous la forme d'une esthétique proprement littéraire, la tâche de l'écrivain étant de trouver non pas les "grands mots ", mais ces " phrases vécues » qui seules peuvent rendre compte avec justesse du monde et rendre une parole aux gens de peu qui en sont privés. Innerhofer n'ignorait pas la difficulté de l'entreprise, notant à propos de l'un de ces laissés-pour-compte :

Wahrscheinlich gebe es im gesamten deutschen Sprachraum keinen Schriftsteller, der Wallisch aufs Papier bringen könne, ohne ihn der Einfachheit halber umbringen zu müssen ${ }^{46}$.

\section{NOTES}

1. Residenz Verlag, Salzbourg, respectivement 1974, 1975, 1977 (titres abrégés dans la suite en $\mathrm{ST}$, SS et GW, et cités dans la réédition de 2002).

2. Ce mode de réception apparaît nettement dans les histoires littéraires et les évaluations d'ensemble de l'œuvre parues lors du suicide de l'écrivain, en 2002, ou lors de la réédition de la trilogie peu après. Voir à ce sujet, par exemple, Viktor Žmegač (dir.) : Geschichte der deutschen Literatur vom 18. Jahrhundert bis zur Gegenwart, Königstein/Ts., Athenäum, 1984, vol. III/2, p. 806 ; Wolfgang Paterno: "Langer Abschied", Profil, Vienne, 28.1.2002, p. 115 ; Karl-Markus Gauß : «Die Verweigerung des Frondienstes. Franz Innerhofers Roman-Trilogie Schöne Tage, Schattseite und Die großen Wörter », Süddeutsche Zeitung, Munich, 28. 2. 2003.

3. On sait la filiation qui peut être établie entre la Heimatdichtung plus ancienne et la Blut- und Bodenliteratur mise à l'honneur par le nazisme. La question se complique, en Autriche, en raison du double rapport de proximité et d'antagonisme entre austro-fascisme et nazisme, l'opposition du Ständestaat à l'Allemagne hitlérienne ayant permis de conserver plus facilement après la guerre une esthétique venue du roman de terroir mise au service de «l'idéologie autrichienne » ( Österreich-Ideologie), notamment dans les manuels scolaires et à travers un certain nombre d'institutions du monde littéraire. Voir à ce sujet, par exemple, Klaus Zeyringer : Österreichische Literatur seit 1945. Überblicke, Einschnitte, Wegmarken, Innsbruck, Haymon Verlag, 2001, p. 65-73.

4. ST, p. 7.

5. «Den Leuten, die auf $48 \mathrm{zu}$ Besuch waren, wurde er meistens als Jugendsünde des Bauern oder als sein Lediger vorgestellt. » (ST, p. 68).

6. Voir l'entretien de Franz Innerhofer avec son ami et futur biographe Frank Tichy, en 1993, où l'écrivain souligne à plusieurs reprises l'importance des figures secondaires et s'élève contre les critiques trop attachées au seul personnage principal, qu'il conçoit plutôt comme un porteparole: «Da waren Leute, die ich erfinden wollte. Franz Innerhofer im Gespräch mit Frank Tichy ", Literatur und Kritik, Salzbourg, Otto Müller Verlag, mars 2002 (la biographie de Frank Tichy est parue sous le titre Franz Innerhofer. Auf der Suche nach dem Menschen, Salzbourg, Residenz Verlag, 2004). Dans un entretien de 1978, Innerhofer soulignait déjà les mêmes enjeux : « Gelebte 
Sätze statt großer Wörter. Ein Gespräch mit dem österreichischen Schriftsteller Franz Innerhofer », Frankfurter Rundschau, Francfort/ Main, 29.3.1978.

7. ST, p. 5.

8. L'arrivée à la ferme paternelle, avec les célèbres trois répliques mises en valeur dans un paragraphe indépendant - «Da gehst her ! Dort bleibst! Ruhig bist !» (ST, p. 12) - en témoigne autant que la première évocation de sermon visant les filles-mères (ibid., p. 7).

9. Le motif des « regards annihilants» («vernichtende Blicke») lancés par les femmes revient sans cesse dans toute la trilogie. La première occurrence concerne l'attitude de la grand-mère de Holl vis-à-vis de sa fille pécheresse (ST, p. 7), puis sa propre mère le punissant ainsi lorsqu'elle ne peut le battre (ibid., p.9) et enfin sa belle-mère à la ferme paternelle. On le retrouvera jusque chez mademoiselle Gärtner, qui parvient par cette arme à désarçonner le jeune homme qu'est devenu Holl dans Die großen Wörter (GW, p. 129-131). Quant à la violence physique, présente dès la première page, elle va s'exacerber chez Rudolf Lein, donnant lieu à des scènes toujours citées en exemple comme celle de la première correction, avec le rituel qui oblige Holl à demander son châtiment et à en remercier ensuite son père (ST, p. 29-30). Le ton est donné dès l'arrivée de Holl dans ce nouveau séjour, où la résistance de l'enfant est brisée en deux semaines par les coups (ibid., p. 12-13).

10. «Aus einer kleinen Welt in eine Welt von Stößen und Schlägen, meistens irgendwo in eine Ecke verbannt, schaute Holl den Frauen zu, die unentwegt kamen und gingen. » (ibid., p. 5).

11. «Da Holl sich noch nichts zusammenreimen konnte, häufig aber Züchtigungen über sich ergehen lassen mußte, weil die Erwachsenen von ihrem Verhalten auf das des Kindes schlossen, begriff er immer weniger. Er saß oft stundenlang hinterm Küchentisch, aß nichts, fragte nichts. Dort hatte er sich so eine Art Heimat gebildet.» (ibid., p. 7) ; «Wie ein Soldat, den Machthaber zwingen, in einem fremden Land unbekannte Leute zu überfallen, ging Holl aus der Wohnküche in die neuen Tage. » (ibid., p. 9).

12. "Ausgeleert wie eine Jauchengrube, ging Holl zu Lichtmeß in der Küche umher. Hätte ihn die Stiefmutter gelassen, er wäre auf die heiße Herdplatte und hätte getanzt. Er verlor ja jedes Mal die ganze Welt. » (ibid., p. 25). Cette scène située à la fin de la première année que passe Holl à la ferme de son père, en février 1951, se répète six ans plus tard, laissant Holl tout aussi désemparé : «Lichtmeß kam. Maria wanderte ins Spital. [...] Neiser zog in die Strafanstalt. Felbertaler zog in die Strafanstalt. Konrad ging auf den Bau. Gufft ging zu einem anderen Bauern. Loferer ging $\mathrm{zu}$ einem kleineren Bauern zurück [...]. Rosa wurde Dienstmädchen in einem sogenannten besseren Haus. Prosch blieb. Moritz wurde nicht gefragt. Holl musste [...] sofort in den Stall [...]. Alles trostlos. Kalt. [...] Für ihn wieder alles hin. » (ibid., p. 182-183).

13. Ibid., p. 29 et passim.

14. «Die ersten Riemenhiebe schmerzten am meisten, dann sah Holl nur noch gelangweilt zum vergitterten Fenster hinaus. Das Keuchen des Vaters widerte ihn an. » (ibid., p. 48). « Der Ekel vor seinem Erzeuger war auch der Ekel vor den Mahlzeiten, die er an der Seite seines Erzeugers einnehmen mußte.» (ibid., p. 54-55). Le thème ne cessera d'être repris, varié et développé dans toute la trilogie.

15. Ibid., p. 57-59.

16. Ibid., p. 69.

17. Voir le premier long passage consacré à la condition des ouvriers agricoles (ibid., p. 24-25) et le commentaire qui accompagne l'évocation des changements d'emploi à la Chandeleur, où il est notamment précisé : «Wenn ein Knecht oder eine Magd wegging, dann auf jeden Fall bis zum anderen Winkel des Dorfes, um alles, was mit dem alten Bauern zusammenhing, radikal abzubrechen, was natürlich nicht ging, denn das alte Jahr hatte sich schon im Kopf niedergelassen. » (ibid., p. 25-26). L'embauche en forêt ou sur un chantier est mentionnée de plus en plus fréquemment à mesure que l'on avance vers la fin des années cinquante, reflétant l'évolution des campagnes autrichiennes qui vont se mécaniser et se dépeupler d'autant. Holl 
évoque cette possibilité qui lui est encore interdite alors qu'il a juste treize ans (ibid., p. 193), puis comme un projet plus concret lorsqu'il atteint seize ans (ibid., p. 217). Le motif est repris au début de Schattseite dans un tableau présentant les mutations de la campagne autrichienne au début des années soixante(SS, p. 5).

18. «Das seien ja ganz schreckliche Dinge, die sie [die Bäuerin] da neben Holl über ihn daherrede. "Wie über einen Steinhaufen wird hier über Menschen gesprochen." (ST, p. 168). Pour l'ensemble du passage sur l'arrivée de Helga à la ferme 48 et la petite révolution que cela provoque, voir ibid., p. 157-175.

19. «Alle, die Besitz und Ansehen hatten, redeten wie der Bauer und gaben überall den Ton an. Was konnte Holl gegen eine solche Sprechweise ausrichten? Er wußte, der Bauer hat ein neues Verfahren gefunden, um weiterhin meinen Körper zu Geld zu machen, um meinen Körper rücksichtslos zu besitzen. Darum ist es immer gegangen. Holl wollte seinen Körper für sich haben, aber wem würde er das jetzt klarmachen?» (ibid., p. 189-190). «In der Nacht zog er oft mit den Knechten schwere Kälber aus den Kühen, und Stunden später saß er schon wieder auf dem Melkstuhl und molk sich einsam durch den Morgen und dachte an sich und andere Menschen, im Gegensatz zum Bauern, der darüber nachdachte, wie er die Menschen zu Geld machen könne. » (ibid., p. 200-201).

20. «Mit dem neuen Melker, Hermann Klein, verstand er sich gut. Der kannte die Bauern in- und auswendig, legte dem Bauern sofort den Kollektivvertrag vor, verlangte seine freien Tage, war Mitglied der Land- und Forstarbeitergewerkschaft und sagte oft, daß er Sozialist sei.» (ibid., p. 218-219).

21. Innerhofer emploie à plusieurs reprises le terme de Leibeigener (par ex. p. 208), que Josef Winkler utilisera plus tard comme titre d'un de ses romans (Der Leibeigene, Francfort/ Main, Suhrkamp, 1987).

22. ST, p. 233-234. L'expression de "camp de concentration paysan », souvent reprise par la critique, a marqué les esprits.

23. «Eine neue Welt türmte sich hoch vor Holl auf.» (ibid., p. 236). «Eine kurze Mopedfahrt. Freundliche Gesichter. Er betrat ein altes Haus [...]. » (ibid., p. 232). « Er rätselte in den Gesichtern herum, fremd und freundlich.» (ibid., p. 233).

24. Ibid., p. 237 et p. 236-237 pour la description de ce nouvel environnement.

25. L'optimisme trompeur qui marque la fin de Schöne Tage a suscité quelques malentendus auprès de la critique, bien résumés par Klaus Kastberger lors de la réédition de la trilogie après la mort d'Innerhofer : « Nach Meinung der Kritik ging es nach Schöne Tage mit Innerhofers Büchern erst graduell und schließlich abrupt bergab. Die Erzählung Der Emporkömmling (1982) und der Roman Um die Wette leben (1993), in denen sich der Autor mit seiner Existenz als Schriftsteller bzw. mit der Weigerung auseinandersetzt, eine solche Existenz zu führen, wurden kaum mit der nötigen Sorgfalt gelesen. Zu massiv stand damals und steht bis heute das Happy End der Schönen Tage im Raum, nämlich die tröstliche Tatsache, dass man sich allein durch die Kraft des Wortes von einer noch so schlimmen Lebensgeschichte emanzipieren kann. [...] Der Gedanke, der in dem Erstling steckt und der in ihm zu einem Mythos wurde, ist zwar verführerisch, aber grundlegend falsch. Die eigene Lebensgeschichte gut beschrieben zu haben, bedeutet noch lange nicht, mit ihr fertig geworden zu sein. Genau das hat Innerhofer in seinen späteren Büchern gesagt. » (Klaus Kastberger : «Franz Innerhofer. Schöne Tage. Schattseite. Die großen Wörter. Franz Innerhofers autobiographische Trilogie », Die Presse, Vienne, 28. 9. 2002).

Karl-Markus Gauß défend un point de vue analogue dans l'introduction à l'entretien de 1993 (op. cit.).

Sur la réception de la trilogie, voir Évelyne Jacquelin : « Du "camp de concentration paysan" au "siège du communisme mondial": la trilogie de Franz Innerhofer entre radicalité critique et recherche utopique », Le texte et l'idée 20, Nancy, Presse universitaire de Nancy, 2005. 
26. Le signe de cet éveil, sur le plan du dispositif narratif, est le passage au récit à la première personne dans Schattseite.

27. SS, p. 87-88.

28. « Keine Einteilung in Werktage und Feiertage, einfach Tage, Zeit. ( (ibid., p. 90). Voir aussi les passages p. 89-90, 94-96 et passim.

29. «[...] und da auf dem Hof wurden schöne lange Gespräche geführt, auf weichen Sitzgelegenheiten, über Dinge, bei denen ich staunte, daß man darüber überhaupt ein Wort verlieren konnte. Aber wir Kinder durften über unsere ärgsten Verzweiflungen nicht berichten, uns wurden die Vorfahren vorgehalten, eine Zeit, in der es darum ging, hart zuzupacken [...]. Ich fühlte mich erleichtert : Das ist also nicht lächerlich, daß ich als Kind immer reden wollte. Das ist nicht jammern. Das ist reden, sich verständigen, sich an der Gemeinschaft beteiligen. Ich hatte mich ja immer beteiligen wollen, oh, ihr verfluchten Bestien, was habt ihr nun mit uns gemacht? Mit Begierde las ich von dieser Welt da oben.» (ibid., p. 96).

30. Dans ces textes écrits d'une seule coulée, sans chapitres, les enchaînements sont très parlants. Ainsi, la première séquence consacrée à la biographie de Napoléon s'achève sur cette remarque : «Nur arbeiten, sagte Helene, macht blind. » (ibid.). Et quelques pages plus loin, après un bref passage consacré aux relations de Helene avec les paysannes victimes de rapports de domination familiaux contre lesquels elles ne veulent cependant rien entreprendre, débute la série des portraits d'employés dont certaines figures comme Maria Wiesinger ou Ernst Wallisch reviendront jusque dans Die großen Wörter (ibid., p. 105-119). Notons aussi que s'intercale entre ces différentes séquences une remarque sur la lecture des Misérables, de Victor Hugo (p. 114-115).

31. «Die Werktagsflucht, an der mich Joseph Bruckmann teilnehmen ließ, war eine durchaus idyllische. » (ibid., p. 201).

32. «Am besten, ich sag dir, was ich mir oft gedacht habe : zwischen dir und mir war sofort eine große Kluft. [...] Bruckmann sagte nichts. Wir reichten uns Wein Wurst Brot Obst dazu. [...] Bruckmann streckte verlegen ein Bein aus : Eine große Kluft, sagte er endlich : Aber Franz, das kann doch nicht stimmen. Wir sind doch immer gut ausgekommen. [...] Ich bin doch kein Unmensch, der nicht mit sich reden läßt. Ein Unmensch bist du sicher nicht, sagte ich und nahm von Bruckmann die Weinflasche entgegen : Aber du bist ein Unternehmer. » (ibid., p. 207-208).

33. "Als wir weitergingen, meinte Joseph Bruckmann, daß ich es im Leben schwer haben würde. Ich fragte, warum. Einen gewissen Respektsabstand muß ein Werktätiger seinem Chef einräumen. Er hielt einen Vortrag vom braven sparsamen strebsamen Arbeiter, der es zu etwas bringt. Ich lachte ihn aus. » (ibid.).

34. "Geradezu aggressiv waren die glücklichen Gesichter, die einen überall von den Plakatwänden herunter anstarrten, immer in Zusammenhang mit Produkten. [...] Die Natur konnte verschmutzen, verstummen und sterben, wie sie wollte, von den Agenturen wurde sie einfach in ihrer ganzen Frische an die Wand geprangert. » (GW, p. 85-86). Le fossé ainsi creusé entre Holl, marqué par le difficile combat contre une nature austère, et ce nouveau milieu de consommateurs urbains à l'heure de la société de loisirs apparait avec une grande netteté dans la longue scène de l'excursion à Haudorf et dans la hutte d'alpage, moment douloureux dans lequel s'enracine aussi un désir d'écrivain : «Wäre er ein episches Erzähltalent gewesen, hätte er sich an den Tisch oder aufs Bett von Bartl [einem früheren Knecht] gesetzt und hätte von einem alten Stiefel oder einem von den vielen in Wände, Tischplatten und Türen geschnitzten Blockbuchstaben und Namen ausgehend, in Ruhe zu berichten angefangen, dann hätten Stecher [ein Student] und seine Freunde nicht gekichert und sich über Lebensumstände, deren erstarrte Vergangenheit sie nun mit Fingerspitzen berührten, lustig gemacht, sondern wären aus der Hütte gewichen.» (ibid., p.118). Voir aussi des développements semblables, mais seulement esquissés p. 43-44.

35. Voir certains portraits de professeurs et d'élèves ainsi que diverses scènes à l'école du soir (ibid., p. 14-16, 47-49, 60-80). 
36. «Die Welt des Redens hatte sich keine Arbeitsgeschichten, keine schweren Geschichten, zu erzählen und fragte deshalb nur nach wenigen Dingen. Ort und Datum der Geburt. Beruf. Name. Eltern. Vorstrafen. Handgeschriebener Lebenslauf. Mehr will die Welt, die vom Reden lebt, von einem Angehörigen, der aus der Arbeitswelt kommt, nicht wissen. Sie steht höchstens da, mustert ihn mit einem kühlen Blick, fragt aber nicht, was ihm denn in der Arbeitswelt zugestoßen sei, dass er nun in die Welt des Redens vordringen wolle. » (ibid., p. 44-45).

37. Ibid., p. 95.

38. Ibid., p. 67, 83 et passim. Des militants apprentis ou chevronnés, Stürzl dit notamment: «Diese jungen Leute sind eine Katastrophe. Kaum haben sie begriffen, daß Unternehmer Ausbeuter sind, verschränken sie schon die Arme über der Brust und lächeln zufrieden, weil ihnen vorkommt, sie wären schon zu einem neuen Verstand gekommen. » (ibid., p. 81).

39. Dans une notice par ailleurs sévère, $W$. Martin Lüdke tente d'établir une relation dialectique entre cette critique de la langue chez Innerhofer et la tradition de la Sprachphilosophie autrichienne («Franz Innerhofer »,in : Kritisches Lexikon zur deutschsprachigen Gegenwartsliteratur, 6/02, version du 1.8.1993, p. 2-3).

40. «Dichter Franz Innerhofer : Vom Hinterwäldler zum Redeweltler », Profil, Vienne, 1. 6. 1977, p. 55.

41. La trilogie évoque notamment en diverses occasions l'entrée des chars soviétiques à Budapest en 1956 et à Prague en 1968, ce dernier événement étant contemporain de l'action dans Die großen Wörter.

42. Ibid., p. 81-82.

43. «Sprache sei ein Transportmittel. Um möglichst viele Bauern zu vereinsamen und in den Selbstmord zu treiben, müsse man die Sprache von ihnen wegtransportieren. Sprache gehöre in die Institutionen, wo die Leute wüßten, wie das Leben $\mathrm{zu}$ funktionieren habe. Die Sprachwissenschaft sei weit fortgeschritten. » (ibid., p. 171).

44. «Von den Revolutionen, sagte Stürzl lachend, ließe sich nun schon ganz gut leben. Er könne, anstatt anstrengende Spaziergänge zu machen, Stücke aus der Revolution vortragen und davon leben.» (ibid., p. 172); «Bestürzt sei er [Stürzl] allerdings manchmal über den Mut der Intellektuellen. Die Intellektuellen seien vor allem sprachlich gut. "Schon Stalin soll ein passabler Sprachwissenschaftler gewesen sein." " (ibid., p. 169).

45. "Er habe sich lange überlegt, ob er das Summerauer Manifest aus der Hand geben sollte. Torberg, sein ehemaliger Zimmerkollege, habe jedenfalls das Summerauer Manifest nicht. An soviel könne er sich erinnern. Er habe das Summerauer Manifest aus der Hand geben müssen. Ziller könnte es haben. Ziller fahre oft in die Sahara. Ziller könne es gelesen und in der Sahara verworfen haben. Es sei auch möglich, daß Holl es an Lorz weitergegeben habe. Aber Lorz sei tot. Lorz sei beim Bergsteigen abgestürzt, habe er von Ziller erfahren. Schließlich log Stürzl noch, daß ihm das Summerauer Manifest zwischen Linz und St. Pölten von einem alten Mann im Zug zugesteckt worden sei. » (ibid., p. 173). Pour l'interprétation plus détaillée de ces dernières pages, voir Évelyne Jacquelin, op. cit..

46. Ibid., p. 170. 


\section{RÉSUMÉS}

La trilogie autobiographique publiée par Franz Innerhofer entre 1974 et 1977 est considérée comme l'un des modèles de la Antiheimatliteratur autrichienne. Le premier volet, Schöne Tage, inverse le thème de l'attachement au pays natal, celui-ci s'imposant à l'individu dans une brutalité muette qui rend impossible tout enracinement dans l'ici. Le mouvement fondamental de la trilogie réside dès lors dans la recherche d'un ailleurs moins hostile, ce qui ne se résume pas à des changements de lieux et de milieux, mais constitue un mode d'appréhension du réel marqué par une double dynamique de rejet et de nouvelle exploration. C'est donc moins l'ascension sociale du personnage central, Holl, qui s'inscrit au premier plan des deux romans suivants, Schattseite et Die großen Wörter, que sa naissance à l'esprit critique. Les horizons successifs vers lesquels il se tourne n'y résistant pas, l'espérance de Holl se trouve renvoyée vers un espace toujours plus lointain et abstrait. Dès lors, la question de l'ailleurs se reformule progressivement en termes politiques: l'épuisement des possibles géographiques, sociaux et culturels débouche sur la recherche d'un « autre part " plus radical, celui de l'utopie. Le tragique propre à la trilogie de Franz Innerhofer réside dans le fait que cette attente ultime ne puisse finalement être prise en charge par aucun discours cohérent, aporie traduite par la désagrégation du texte lui-même sur laquelle s'achève une œuvre qui propose dans le même mouvement l'esquisse d'une esthétique littéraire particulièrement exigeante.

Die von Franz Innerhofer zwischen 1974 und 1977 veröffentlichte autobiographische Trilogie wird als ein Musterbeispiel österreichischer Antiheimatliteratur angesehen. Im ersten Teil, Schöne Tage, wird das Thema der Heimatverbundenheit umgekehrt: die angeblich vertraute Umwelt zwingt sich dem Einzelnen in stummer Brutalität auf, so dass jede Verwurzelung im Hier unmöglich wird. Die Grundbewegung der Trilogie besteht also in der Suche nach einem weniger unwirtlichen anderen Raum, was aber nicht auf Orts- und Milieuwechsel begrenzt bleibt, sondern sich auch als ein Modus der Realitätswahrnehmung erweist, der durch eine doppelte Dynamik von Ablehnung und weiterer Erforschung gekennzeichnet ist. Demnach steht weniger der soziale Aufstieg Holls, der zentralen Figur, im Vordergrund der beiden folgenden Romane, Schattseite und Die großen Wörter, als dessen Entwicklung zum kritischen Geist. Die verschiedenen Kreise, zu denen er sich sukzessiv hinwendet, halten diesem Geist nicht stand, so dass Holls Hoffnung in einen immer ferneren und abstrakteren Raum rückt. Auf diese Weise wird die Problematik des anderen Raums allmählich auf politischer Ebene formuliert: Die Ausschöpfung der geographischen, sozialen und kulturellen Möglichkeiten führt zur Suche nach einer radikaleren Form des Woanders, der der Utopie. Die der Trilogie Franz Innerhofers innewohnende Tragik besteht darin, dass dieser letzten Erwartung schließlich kein kohärenter Diskurs Rechnung tragen kann, eine Aporie, die am Ende des Werkes im Auseinanderfallen des Textes selbst ihren Ausdruck findet, wobei gleichzeitig eine besonders anspruchsvolle literarische Ästhetik skizziert wird.

The autobiographical trilogy published by Franz Innerhofer between 1974 and 1977 is considered as one of the models of the Austrian Antiheimatliteratur (anti-regionalist literature). The first wing Schöne Tage (Beautiful Days) reverses the theme of the attachment to the homeland, this one imposing itself to the individual in a mute brutality that makes impossible any deep-rootedness in the here. The fundamental movement of the trilogy lies then in the search of an elsewhere that would be less hostile, which does not come down to changes of places and times but constitutes a mode of apprehension of the real marked by a double dynamic of reject and new exploration. So, it is less the social ascension of the main character, Holl that is placed in the foreground of the two following novels: Schattseite and Die großen Wörter than his birth to the 
critical spirit. As the successive horizons he is turning to cannot resist it, Holl's hope is taken back to a space that is still more in the distance and abstract. From then on, the question of the elsewhere is progressively to be asked in political terms: exhaustion of the geographical, social and cultural possible leads to the search of "another part", that is more radical, the one of utopia. The tragic that is proper to Franz Innerhofer's trilogy lies in the fact that this ultimate action cannot eventually be taken into account by any coherent discourse, an aporia expressed by the of the text itself on which a work comes to an end and that offers in the same movement the sketch of a particularly demanding literary aesthetics.

\section{INDEX}

oeuvrecitee Die großen Wörter, Schattseite, Schöne Tage

Mots-clés : Anti-Heimatliteratur

\section{AUTEURS}

\section{EVELYNE JACQUELIN}

Université d'Artois 